\title{
Maps and the Internet: What a Mess It Is and How To Fix It
}

Michael Peterson Department of Geography/ Geology University of Nebraska at Omaha mpeterson@mail.unomaha.edu
The promise of the Internet for cartography has faded into stark realities of commercialism, connectivity problems and confusion about what represents quality in Internet mapping. Accessing the Internet is still problematic and a great digital divide separates the developed from the developing world. Interaction with the online map, the single greatest advantage of maps and the new medium, has been either poorly implemented or not incorporated at all. The commercial aspect of the Internet has been turned upside down. We pay to access the Internet, not for its content. As a result, there is little competition to improve the quality of online maps, other than for bragging rights, and little incentive to create quality content. On top of this, in many parts of the world, access to the Internet by computer is expensive or inconvenient and people prefer to use the Internet through their mobile phone. Almost all new users to the Internet are connecting through mobile devices and a small screen that is hardly suitable for the display of maps. While a de-centralized system like the Internet is impossible to fix in traditional ways, solutions must be found for making the medium more accessible and useful for maps. National and international organizations can play a key role in providing examples of what is possible with maps and the Internet. Low-cost, easy-to-use tools also need to be made available so that online cartographers can create quality content.

Begun in the late 1960s as an experiment in failsafe file exchange between computers, the Internet has evolved into a fascinating, if problematic, worldwide communications medium. The incorporation of the World Wide Web protocol in the early 1990s dramatically expanded its use. According to the Internet WorldStats web site, it is now estimated that one sixth of the human population uses the Internet on a regular basis (2007). Some estimates put the daily page count at over 47 trillion (Rangarajan 2007).

The expansion of the mobile Internet through cell phones has been particularly astounding since 2000, but delivering content is still in the early stages of development. Cell phone companies have entered into agreements with search providers like Google and Yahoo, thus acknowledging the need to make the use of the Internet similar between desktop computers and cell phones (Rangarajan 2007). Analysis of consumer usage for one mobile Internet service in other countries shows that user habits are similar to desktop users'. The data also shows that, even though consumers face an initial orientation hurdle using the Internet on a small screen without a keyboard and mouse, once they adapt, their usage grows steadily month after month (Rangarajan 2007). The level of Internet use through cell phones in the United States is much lower than in many other countries.

Maps represent a major component of Internet traffic. Common web mapping sites, including MapQuest, Yahoo, and Google Maps, each report making millions of maps per day. According to ComScore Media Matrix, AOL's Mapquest had 45.1 million US visitors during February 2007, Yahoo 
had 29.1 million users, and Google maps had 22.2 million U.S. visitors (Liedtke 2007). People now look to the Internet to find all manner of maps, and it has clearly become the new medium for cartography.

But, this new medium is not without its problems. The security and reliability of the Internet is increasingly under attack. Some warn of a digital Armageddon brought on by spammers, hackers, phishers and cyberterrorists. They argue that the Internet is "at the tipping point of overwhelming abuse and complexity" (Anthes 2007). In addition, the neutrality of the Internet is being challenged by Internet Service Providers (ISPs) that want to restrict access to competing sites. Many countries place restrictions on Internet access for political purposes. In less-developed and moderately developed parts of the world, the use of the Internet is beyond the financial means of most people, contributing to a great "digital divide." Most new users of the Internet are accessing its resources with the tiny screen of mobile phones, much different from desktop computer systems that, in contrast, are using increasingly larger screens.

While map use has expanded rapidly with the new medium, the quality of Internet maps has not evolved appreciably over the last decade. Attempts to introduce higher quality, vector-based maps have not progressed beyond experimentation. The high cost of developing and maintaining map servers has become a stark reality for many map providers, and the lack of a revenue stream for content providers makes it increasingly difficult to both provide content and maintain servers.

Clearly, all is not well in the world of maps and the Internet. This paper examines the major problems associated with the Internet and Internet maps.

\section{Problems with Internet Access}

In contrast to what we might like to believe, the Internet is not a free and open system of data communications. The constraints that limit people's access to the Internet include governmental restrictions, business decisions, and the costs of hardware, software, and connectivity. In addition, the mobility requirements of the user make it difficult to maintain a connection. Other access problems include language barriers, users' varied educational levels, and the general complexity of the system.

Sometime during 2006, the Internet added its 1 billionth user. As Rezwan (2007) points out, adding the next billion will be a major challenge. The Internet is becoming more fragmented, and international borders are increasingly visible. The large gap between rich and poor is apparent by the level of Internet access. The notion of a free, common, global Internet that can unite the world is merely an illusion.

One of the most vexing problems with the Internet is the cost of access. In addition to hardware requirements, monthly fees for Internet access can strain most budgets. In developed countries, people forfeit their traditional telephone and television cable connections to pay for Internet access. In the developing parts of the world, telephone and television cable connections have yet to be installed, so there is no money to save by switching services.

\section{Global Digital Divide}

The developed nations are benefiting enormously from the information age while developing nations are struggling to keep pace. This difference in technological progress is widening the economic disparity between the most developed nations of the world (primarily Canada, the United States, 
Japan, South Korea, Western Europe and Australasia) and developing ones (primarily Latin America, Africa, and Southeast Asia), thus creating a digitally fostered divide ( $\mathrm{Lu}$ 2001). Unlike the traditional notion of the "digital divide" between social classes, the "global digital divide" is essentially a geographical division. Figure 1 shows the percentage of Internet users by country.

Many see mobile devices as the great equalizer of the digital divide. The PC, for most of the world's poor, is too expensive, too complicated and needs more power. The mobile phone is far more ubiquitous, and stories are emerging from developing countries of how the new communication devices are helping farmers find better prices for their agricultural products.

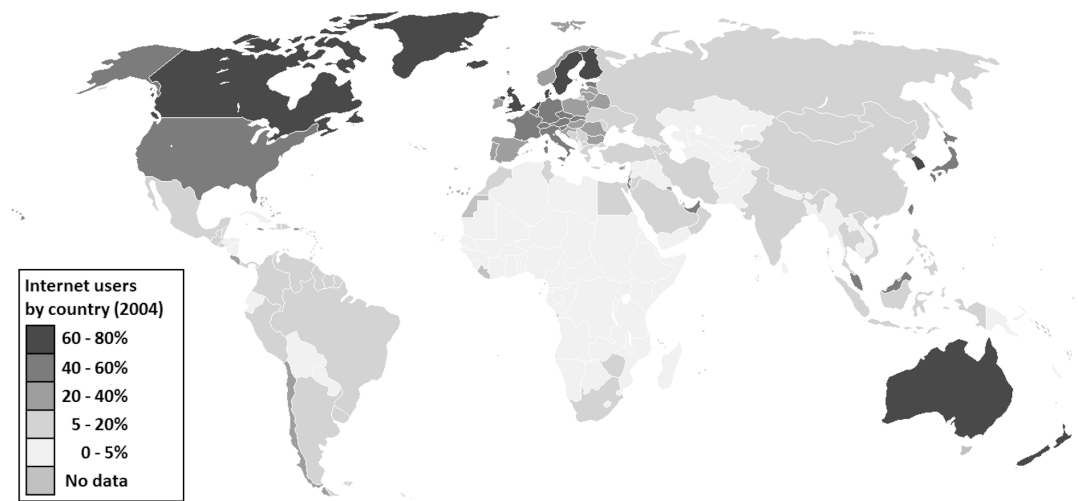

http://upload.wikimedia.org/wikipedia/commons/5/5b/Internet_users_en.png

Figure 1. The percentage of Internet users by country. (see page 67 for color version)

\section{Governmental Restrictions on Internet Access}

Many governments are becoming increasingly concerned about the potentially de-stabilizing role of the Internet. In particular, the new social networking aspect of the Internet can unite groups of people in dissent or protest. Governmental efforts are increasing in some countries to limit access to the Internet or specific Internet sites. Two examples are noted here.

The relationship between the largest Internet company, Google, and the largest country in the world, China, is noted for its pragmatism. Google recognizes the size of China's Internet market and China recognizes the power of Google's online enterprise. Google has so far escaped from being banned in China by agreeing to limit what is displayed as a result of certain Internet searches through Google's search engine. The results of a Google.cn search are filtered by people working for Google in California so as not to bring up any results concerning the Tiananmen Square protests of 1989, sites supporting the independence movements of Tibet and Taiwan or the Falun Gong, and other information perceived to be harmful to the People's Republic of China (Wikipedia 2007).

The second example comes from Belarus. In February 2007, Belarusian authorities expanded restrictions on Internet usage, requiring owners of Internet cafes and computer clubs to keep logs of Web sites accessed by users and report them to security services. Internet usage is already subject to restrictions in Belarus (Associated Press 2007). Citizens must present identification documents to use Internet cafes, and Internet access for offices and private users is controlled by a state monopoly. Criticizing President Alexander Lukashenko and other senior government officials remains a criminal offense in Belarus. 


\section{Net Neutrality}

Net neutrality is the principle that Internet users can go to any website, run any web application, and attach any device to the network without restriction by the Internet service provider. Two companies in the United States, AT\&T and BellSouth, have proposed a high-speed broadband network that would be separate from the public Internet, providing its own video service at a guaranteed level of quality. The concern is that these broadband providers will create a fast Internet for their own services - at a premium price - and a slow lane for everyone else. A premium Internet service might also effectively impose a class structure for the control of spam or online security (Bicknell 2006a).

Vint Cerf, chairman of Icann and co-creator of the TCP/IP standard, warns against creating a two-tier web system. He believes that "the remarkable social impact and economic success of the Internet is directly attributable to the architectural characteristics that were part of its design" (Bicknell 2006b). The Internet was designed with no gatekeepers over new content or services, an end-to-end model that allows people at each point on the network to innovate free of any central control (Bicknell 2006b).

\section{A New Internet}

All of the technical problems inherent in the Internet have prompted some to propose an entirely new Internet that specifically addresses the security and privacy issues. A group of computer scientists at Stanford University argue that complexity is crushing the Internet. They point out that the original Internet design was based on the idea that users were immobile and connected by wires. This is no longer the case (Casado et al. 2007).

The group proposes a prototype network that centralizes security rather than placing it around the network in firewalls or in client-based virus detection programs. In his prototype, all communications are turned off by default. A host joining the network must get explicit permission from a centralized server before it can connect to anything except that server. In addition, the server won't grant permission unless it is able to determine the location and identity of the requestor (Casado et al. 2007). The proposed centralized server acts as an administrator and essentially monitors all computers connected to the Internet. Such a system would be in sharp contrast to the existing Internet that was specifically designed to not be dependent upon a centralized server. Destroying the server could easily stop an Internet based on a centralized server.

\section{Internet Addiction}

Finally, the Internet is leading to social problems. Specifically, there is concern about excessive Internet use by some people-variously termed Internet addiction, problematic Internet use, pathological Internet use, and compulsive Internet use. There is no consensus on how to diagnose the problem in individuals, but there is agreement that some people are overdoing the amount of time they spend on the Internet (Payne 2006). The problem is getting more serious attention as the use of the Web grows. According to a 2005 survey, Internet users average about 3.5 hours online each day (Payne 2006). Rather than using the term "addiction," Yellowlees and Marks (2007) simply define a class of individuals as having problematic Internet use (2007). These include people who have a history of impulse control and addictive disorders. The American Psychiatric As- 
sociation is considering listing Internet addiction in the next edition of its diagnostic manual (Payne 2006).

\section{Problems with Internet Maps}

The problems addressed to this point deal with the Internet in general. A whole series of other additional problems can be identified with Internet maps.

\section{Accuracy}

An incident in California during December 2006 has brought the accuracy of online maps under public scrutiny. While traveling in northern California, the Kim family from San Francisco turned onto a small logging road. After becoming stranded on the road during a snowstorm, the husband walked for help and died shortly before his family was found by a search party. The road is normally impassable in winter-a fact well known to locals-but Google Maps, Live Local, and Ask.com recommends the route (Fulbright 2006). The incident brought public warnings about Internet maps. A number of problematic routing examples were subsequently cited with routes that appear to be a shortcut but are seasonal or dangerous-or routes that contain outright errors. The problem is made worse through the use of turn-by-turn directions that are offered by the Internet mapping sites. Many users prefer these directions to the associated maps, effectively leaving them lost when they deviate from the written directions.

\section{Maintaining Servers}

In contrast to the finality of printing a map, the work of maintaining an Internet map server is never complete. New data or new Internet protocols make it necessary to make continual updates to a server. A case in point is the difficulty of maintaining the US Census Bureau Tiger Map Server ("TMS") (US Census 2007).

The TMS system came online in 1995 "to demonstrate cost efficient delivery of public data and research and development of the Census Bureau applications on the Internet" (US Census 2007). It has been operating since on two Silicon Graphics servers, each with 200MB of RAM and 9GB SCSI2 disk drives - miniscule numbers by today's standards. The server is still in operation thirteen years later, but no contingency has been made to transfer the server software or the data to another computer. When these computers fail, the system will cease. There are many map servers in different parts of the world that cannot be upgraded or migrated to another computer and will soon fade away.

\section{The Google Maps Effect}

Google Maps was introduced in 2005 and has revolutionized online mapping. Implementing a new server/client system called AJAX, Google Maps increases the level of interaction between the user's computer and the map server. Panning is accomplished effortlessly by moving the mouse from side-to-side, and the scroll button can be used to zoom in and out. Map updates are almost instantaneous. Combining maps and satellite imagery, the stand-alone Google Earth application also has a devoted user-base.

The Google interface has transformed online mapping and left other sites seeming instantly inferior. Once a map user has used the Google Map 
interface, they don't want to use any other type of interactive map. Essentially, Google Map has eclipsed ten years of work in server/client interactive mapping that was based mostly on the server constructing a map in raster format and embedding this into a web page that was returned to the user. A typical reaction by many to this older form of interactive online mapping is, "Why can't this site be like Google Maps?" Google Maps also allows users to enter their own information onto the map that can be shared with other users (Liedtke 2007), and its Application Programming Interface (API) allows programmers to construct their own maps. Of course, the hidden secret of Google Maps is that it uses the Mercator projection. The scale varies constantly as the map is moved to the north or south.

Any new technology will naturally involve a considerable amount of experimentation. But, in the case of online mapping and web-based GIS, a great deal of money and effort has been expended on creating interactive mapping sites. Converting these to the new Google Map standard will require a great deal of effort because of the new way that the map is transferred to the user's computer. Switching to the new AJAX method for online map presentation will be time-consuming and expensive.

\section{Mobile Mapping}

The main application of mapping for mobile devices is navigation assistance or wayfinding. While the primary purpose of wayfinding with maps is to get to the destination with as little effort as possible, the secondary purpose is the creation of a mental map of the route that will aid in finding the location again without the use of a map. In other words, the purpose of the map in wayfinding is to create a mental construct such the map will be rendered meaningless when the same task is performed again. The map succeeds by becoming irrelevant.

In contrast, when using a mobile device for wayfinding, the user is directed to a location with minimal mental effort by the user. In addition, the schematic depictions presented on the mobile device are often too simplistic to create a functional mental map of the environment. Because there is little overlap between the map and the environment, the quality of the resultant mental map is compromised. It is very likely that the user will need to get instructions from the device again for not only the return trip but for any future trip to the same location. The mobile device has succeeded by creating a permanent dependence on the device.

Being told where you are bypasses the process of finding out where you are, thus hindering the formation of mental maps. Finding out where you are helps to form a mental map, a mental conception of where you have been and where you need to go. Mobile mapping devices, like navigation systems in general, do not seem to contribute to the formation of long-term mental maps.

\section{The Open Source Dilemma}

A variety of open source software projects have had a major impact on all forms of computing, especially server-based applications. A prime example is Apache, the main application in use for web servers. Open source online mapping applications are also in widespread use-the main example being MapServer.

While open source software is "free," installing and using the software is complicated and time-consuming. Creating a simple-to-use installation procedure and application user interface is not a primary focus for open 
source developers. The user interface is often left to the person who is installing the software. Updating open source software is also complicated. So, while the software is free, one must deal with cumbersome interfaces and less than appealing online mapping sites. MapServer sites, and there are hundreds of these around the world, mostly implement a non-Google map interface that many users now find frustrating.

Open source developers have also given little attention to improving the graphic quality of the maps themselves. While the software provides a more feasible and cost-effective approach to implement online mapping, the movement is designed primarily for programmers and suffers from not easily allowing input from a broad range of individuals who could make non-programming type of improvements. In short, open source is only open to programmers. A broader developer- and user-community could have a major influence in creating high-quality online mapping sites.

\section{Solutions and Summary}

With all of its problems, the Internet remains an amazing communications system. New applications are continually introduced and new users are joining the system every day. Because the Internet is an unmanaged, non-centralized system, a central authority cannot fix it. Internet users and organizations of users will define the future Internet.

While the development of Internet cartography has been at least as significant as that of the printed map, a considerable amount of effort is still required to make the new medium a truly effective and useful means of conveying and analyzing spatial information in the form of maps. Hundreds of millions of map users have been introduced to interactive maps through the Internet. Online interactive mapping sites represent how most younger people have learned to use maps. New mapping sites, such as Google Maps, are quickly embraced by Internet users looking for new ways to map the world. The mass appeal of the Google Earth product is another indication of the public's desire to interact with maps and satellite images in new and exciting ways.

National and international organizations like NACIS and the International Cartographic Association (ICA) have a major role to play in defining the function and form of Internet maps. Online map galleries sponsored by the organizations could be used to highlight innovative map displays. Cooperation and active participation with open source efforts can lead to meaningful improvements in this type of software. Hands-on workshops and seminars, such as those conducted by the Maps and the Internet Commission of the ICA, are a valuable way of conveying advances in technology to a broad and diverse audience. Although we might lament the passing of the paper era in cartography, it is necessary to embrace the changes that the Internet brings to the discipline and seek improvements in the science and technology of a new Internet cartography. ary 8 <http://www.computerworld.com/action/article.do?command=vi ewArticleBasic\&articleId=279934\&intsrc=hm_ts_head $>$

Associated Press Worldstream. 2007. Belarus imposes tougher restrictions on Internet use, February 15. 
Bicknell, David. 2006a. Internet at a crossroads. Computer Weekly, November 14. <http:/ / www.computerweekly.com/Articles/2006/11/09/219800/internet-at-a-crossroads.htm>

Bicknell, David. 2006b. The end of the web as we know it? Computer Weekly, November. 28. <http://www.computerweekly.com/Articles/2006/11/23/220129/the-end-of-the-web-as-we-know-it.htm>

Fulbright, Leslie. 2006. A family's tragedy: Internet travel directions need to be checked carefully. San Francisco Chronicle, December 7. <http:// www.sfgate.com/cgi-bin/article.cgi?f=/c/a/2006/12/07/MNGTMMQTVO1.DTL>

Internet World Stats. 2007. Internet Usage Statistics. <http:/ / www.internetworldstats.com/stats.htm>

Liedtke, Michael. 2007. Google turns to users for online maps. Associated Press Online, April 5. < http://www.ap.org/>

Lu, Ming-te. 2001. Digital divide in developing countries. Journal of Global Information Technology Management 4 (3): 1-4.

Casado, M., et al. 2007. Ethane: Taking control of the Enterprise ACM SIGCOMM '07, August, Kyoto, Japan. <http://www.sigcomm.org/ccr/ drupal/?q=node/242>

Payne, J. W. 2006. Caught in the Web: More people say heavy Internet use is disrupting their lives, and medical experts are paying attention. The Washington Post, November 14. <http:/ / www.washingtonpost.com/wpdyn/content/article/2006/11/10/AR2006111001571.html>

Rangarajan, Jayanthi. 2007. Mobile creates increasingly pervasive Internet. Total Telecom, January. 16. <http://www.totaltele.com/View. aspx?t=4\&ID=89070>

Rezwan, 2007. Globalization of Internet: The next billion users are coming with a challenge. The 3rd World View, January 26. < http:/ / rezwanul. blogspot.com/2007/01/globalization-of-internet-next-billion.html>

US Census Bureau. 2007. Tiger mapping service: The "Coast-to-coast" digital map database. <http:/ / tiger.census.gov/credits.html>

Yellowlees, Peter M., and Shayna Marks. 2007. Problematic Internet use or Internet addiction. Computers and Human Behavior 23.3 (May), 1447-1553.

Wikipedia. 2007. Censorship by Google. <http://en.wikipedia.org/ wiki/Censorship_by_Google> 